\title{
PREVALÊNCIA DE QUEDAS EM IDOSOS NA COMUNIDADE
}

\section{Heloísa Silva Guerra}

Fisioterapeuta, Mestre, Docente Faculdade de Enfermagem da Universidade Estadual de Goiás (UEG), Campus Ceres e Faculdade de Medicina da Universidade de Rio Verde (UniRV), Campus Aparecida de Goiânia, Brasil.

E-mail: heloisasguerra@gmail.com

\section{Renata Alves e Sousa \\ Daniela Cristina Ferreira Bernardes \\ Juliana Alves Santana \\ Luana Marchese Barreira}

Acadêmicos da Faculdade de Medicina da Universidade de Rio Verde (UniRV), Campus Aparecida de Goiânia, Brasil.
RESUMO: A ocorrência de quedas constitui uma das principais causas de morbidade e mortalidade na população idosa, tendo se tornado um problema importante de saúde pública devido aos altos custos sociais e financeiros que acarreta. $\mathrm{O}$ objetivo deste estudo foi determinar a prevalência de quedas em idosos vinculados a uma unidade básica de saúde. Trata-se de estudo descritivo transversal com a avaliação de idosos a partir de dois instrumentos: um relacionado aos dados sóciodemográficos e outro sobre a ocorrência de quedas nos últimos 12 meses. Os dados foram processados e analisados por meio do SPSS ${ }^{\circledR}$ versão 20.0 para Windows. No total, 97 idosos com média de idade de 70,5 anos ( \pm 8 anos) participaram do estudo 32 idosos referiram ter caído pelo menos uma vez nos últimos 12 meses, estimando prevalência de 33\% (32/97; IC 95\%: 24,2\% - 42,8\%) de quedas entre os participantes. $O$ número de quedas variou de uma a 10 vezes no último ano, com média de duas quedas por idoso ( \pm 2 quedas). A queda da própria altura $(87,5 \%)$ e ocorrida fora do domicílio $(31,3 \%)$, foi a mais referida. $O$ medo de cair novamente foi a consequência permanente da queda mais apontada pelos idosos. A prevalência de quedas neste estudo apresentou índices importantes, trazendo repercussões negativas para a saúde dos indivíduos.

PALAVRAS-CHAVE: Acidentes por quedas; Idoso, Atenção primária à saúde.

\section{PREVALENCE OF FALLS BY THE ELDERLY IN A HOME COMMUNITY}

ABSTRACT: Falls are the main causes of morbidity and death in elderly people. They are an important issue in public health due to high social and financial costs. Current descriptive and transversal analysis deals with the prevalence of falls in the elderly linked to a health unit. It also evaluates them through socio-demographic data and the occurrence of falls during the previous 12 months. Data were processed and analyzed by SPSS ${ }^{\circledR} 20,0$ for Windows. Further, 97 elderly people, averaging 70,5 years $(+8$ years) participated, of whom 32 suffered at least one fall during the last 12 months, with a prevalence of $33 \%$ (32/97; IC 95\%: $24,2 \%-42,8 \%)$ among the participants. The number of falls ranged between one and 10 during the previous year, averaging two falls per elderly ( +2 falls). Falls from one's height $(87,5 \%)$ and outside one's home $(31,3 \%)$ were the most common. Fear of falling once more was a permanent consequence indicated by the elderly. Prevalence of falls in current analysis showed important indexes with negative causes on individual health.

KEY WORDS: Accidental falls; Aged; Primary health care. 


\section{INTRODUÇão}

O envelhecimento populacional é um fenômeno que tem ocorrido mundialmente, caracterizando-se como um dos eventos mais expressivos da sociedade, em particular nos países em desenvolvimento como o Brasil (REZENDE; CARRILLO; SEBASTIÃO, 2012).

Concomitante a esse processo demográfico, mudanças nos padrões de adoecimento e morte têm ocorrido, evidenciando o aumento das doenças crônicas não transmissíveis e das quedas, o que pode gerar incapacidade funcional e diminuição da qualidade de vida nos idosos (SANTOS et al., 2015).

A ocorrência de quedas constitui uma das principais causas de morbidade e mortalidade por causas externas na população idosa, tendo se tornado um problema importante de saúde pública devido aos altos custos sociais e financeiros decorrentes delas, além de ser considerada marcador de fragilidade e de declínio da saúde (PINHO et al., 2012). Pode-se definir queda como sendo um deslocamento não intencional do corpo para um nível inferior à posição inicial, sem correção em tempo hábil e, determinada por circunstâncias multifatoriais que comprometem a estabilidade do indivíduo (SIQUEIRA et al., 2007).

Geralmente as quedas ocorrem no ambiente domiciliar, no momento em que o idoso realiza suas atividades cotidianas, como por exemplo ir ao banheiro, subir e descer escadas ou trabalhar nas tarefas domésticas, sendo que as fraturas são a consequência mais comum resultante deste evento (PINHO et al., 2012). Estima-se que as quedas tenham uma frequência de $75 \%$ em pessoas na faixa etária dos 65 a 74 anos e de 90\% nos idosos com idade superior a 75 anos (PALMA, 2012).

Acidentes como as quedas acarretam fortes encargos financeiros em função das internações, tratamentos e cuidados de urgência que exigem, além de um impacto negativo ao indivíduo e à sociedade (FHON et al., 2013). Conhecer o perfil epidemiológico dessa população e os principais problemas a que estão sujeitos, pode colaborar para o planejamento de ações de saúde mais específicas e subsidiar políticas públicas de atenção ao idoso, que contemplem aspectos preventivos no tocante às quedas.
Este estudo teve como objetivo determinar a prevalência de quedas em idosos vinculados a uma unidade básica de saúde.

\section{METODOLOGIA}

Trata-se de um estudo de caráter descritivo e transversal, realizado em uma unidade básica de saúde da rede pública municipal de atenção à saúde do município de Aparecida de Goiânia, Goiás.

A amostra foi composta, por conveniência, de 97 idosos vinculados à unidade básica de saúde (UBS) Santa Luzia. Os critérios de inclusão foram: idade $\geq 60$ anos e ser residente em área adscrita da UBS em questão. Os idosos que apresentavam algum déficit cognitivo, de acordo com informações dos prontuários, e que utilizavam algum tipo de dispositivo auxiliar de marcha ou cadeira de rodas, foram excluídos.

A coleta de dados foi realizada pelas pesquisadoras no domicílio do idoso por meio de entrevista, em uma única visita.

Para operacionalização da coleta de dados foram utilizados dois instrumentos, sendo o primeiro relacionado aos dados sócio demográficos, com perguntas relacionadas aos aspectos sexo, idade, estado conjugal, escolaridade, renda, ocupação atual, entre outros; e morbidades referidas pelos idosos; e o segundo referente às quedas.

$\mathrm{O}$ instrumento de quedas foi adaptado do estudo de Shiaveto (2008), que o elaborou após revisão bibliográfica nas áreas de gerontologia, geriatria e quedas, e é composto dos seguintes itens de avaliação de quedas: número de quedas nos últimos 12 meses, local em que caiu, se havia ingerido bebidas alcoólicas ou algum tipo de medicamento antes de cair, causa da queda, tipo de consequência, local de fratura (se houve) e resultado da consequência gerada pela queda.

A análise dos dados foi realizada mediante a estatística descritiva, por meio do Software Statistical Packpage for the Social Sciences (SPSS), versão 20.0 para Windows, apresentando as frequências relativa e absoluta para as variáveis categóricas. Calculou-se a prevalência de queda entre os idosos e intervalo de $95 \%$ de confiança, considerando o número total de idosos investigados 
como denominador e o número de idosos que sofreram queda como numerador.

A pesquisa foi aprovada pelo Comitê de Ética em Pesquisa da Fundação de Ensino Superior de Rio Verde - FESURV - Universidade de Rio Verde com Parecer $n^{\circ}$ 1071894 (CAAE 44771715.0.0000.5077), em conformidade com a Resolução no 466/12 do Conselho Nacional de Saúde. A todos os participantes do estudo foi garantida a participação voluntária mediante leitura e assinatura do Termo de Consentimento Livre e Esclarecido - TCLE.

\section{RESULTADOS}

Participaram do estudo 97 idosos atendidos em uma unidade de atenção primária do município de Aparecida de Goiânia, com idade variando de 60 a 95 anos, média de 70,5 anos ( \pm 8 anos), sendo que 48 (49,5\%) estavam na faixa etária de 60 a 69 anos. Na tabela 1 está descrito o perfil sóciodemográfico dos indivíduos, onde observou-se que a maioria correspondia ao sexo feminino, com $61(62,9 \%)$ entrevistadas.

Tabela 1. Perfil sociodemográfico dos idosos atendidos na unidade de atenção primária de um município do estado de Goiás, 2015

\begin{tabular}{lcc}
\multicolumn{1}{c}{ Características } & N & (continua) \\
\hline Sexo & & \\
\hline Feminino & 61 & 62,9 \\
Masculino & 36 & 37,1 \\
\hline Idade & & \\
\hline 60 a 69 anos & 48 & 49,5 \\
70 a 79 anos & 35 & 36,1 \\
80 ou mais anos & 14 & 14,5 \\
\hline Estado civil & & \\
\hline Solteiro & 9 & 9,3 \\
Casado/vive maritalmente & 39 & 40,2 \\
Viúvo & 38 & 39,2 \\
$\quad$ Divorciado & 11 & 11,3 \\
\hline Escolaridade & & \\
\hline Primário & 50 & 51,5 \\
Ginásio/1 ${ }^{\circ}$ grau & 24 & 24,7 \\
Segundo grau & 9 & 9,3 \\
Curso superior & 1 & 1,0 \\
\hline
\end{tabular}

\begin{tabular}{lcc} 
& & (conclusão) \\
\hline \multicolumn{1}{c}{ Características } & N & \% \\
\hline Nenhum & 13 & 13,4 \\
\hline Pessoas com quem reside & & \\
\hline Sozinho & 32 & 33,0 \\
Filhos & 23 & 23,7 \\
Companheiro & 35 & 36,1 \\
Filhos e companheiro & 2 & 2,1 \\
Outra pessoa & 5 & 5,2 \\
\hline
\end{tabular}

\section{Atividades exercidas durante a} vida

\begin{tabular}{lcc} 
vida & 54 & 55,7 \\
Atividade profissional & 23 & 23,7 \\
Serviços domésticos & 20 & 20,6 \\
Outras & & \\
\hline Ocupação atual & 68 & 70,1 \\
\hline Aposentado & 2 & 2,1 \\
Doméstica & 27 & 27,8 \\
Outra & & \\
\hline
\end{tabular}

\section{Renda mensal*}

\begin{tabular}{lcc} 
Nenhuma & 1 & 1,0 \\
Um salário mínimo & 64 & 66,0 \\
Dois salários mínimos & 25 & 25,8 \\
Três a cinco salários mínimos & 6 & 6,2 \\
Cinco ou mais salários mínimos & 1 & 1,0 \\
\hline
\end{tabular}

Tipo de moradia

\begin{tabular}{lcc}
\hline Própria & 77 & 79,4 \\
Alugada & 5 & 5,2 \\
Dos filhos & 2 & 2,1 \\
Outra & 13 & 13,4 \\
\hline Total & 97 & 100 \\
\hline
\end{tabular}

Nota: * Salário mínimo $\mathrm{R} \$ 880,00$.

Fonte: Dados da pesquisa

Em relação ao estado civil 39 (40,2\%) eram casados/viviam maritalmente e 35 (36,1\%) moravam com seus companheiros. Quanto à vida econômica e moradia, 68 (70,1\%) eram aposentados e $77(79,4 \%)$ residiam em casa própria.

A tabela 2 apresenta os hábitos de vida e a situação de saúde atual dos participantes. No tocante aos hábitos de vida, a maioria relatou não fazer uso de bebidas alcoólicas e tabaco, correspondendo a 94 (96,9\%) e 86 $(88,7 \%)$ casos, respectivamente. Dentre os entrevistados, $60(61,9 \%)$ referiram não praticar exercício físico de forma regular e $92(94,8 \%)$ relataram a presença de algum problema de saúde. 
Tabela 2. Hábitos de vida e situação de saúde atual dos idosos atendidos na unidade de atenção primária de um município do estado de Goiás, 2015

\begin{tabular}{|c|c|c|}
\hline Características & $\mathbf{N}$ & $\%$ \\
\hline \multicolumn{3}{|l|}{ Alcoolista } \\
\hline Sim & 3 & 3,1 \\
\hline Não & 94 & 96,9 \\
\hline \multicolumn{3}{|l|}{ Tabagista } \\
\hline Sim & 11 & 11,3 \\
\hline Não & 86 & 88,7 \\
\hline
\end{tabular}

\begin{tabular}{lll}
\hline Prática regular de exercício físico & & \\
\hline Sim & 37 & 38,1 \\
Não & 60 & 61,9 \\
\hline
\end{tabular}

\begin{tabular}{lcc}
\hline Refere problemas de saúde & & \\
\hline Sim & 92 & 94,8 \\
Não & 5 & 5,2 \\
\hline
\end{tabular}

\begin{tabular}{|c|c|c|}
\hline Doenças referidas & & \\
\hline Visão prejudicada & 60 & 61,9 \\
\hline Hipertensão Arterial & 60 & 61,9 \\
\hline Problemas de coluna & 60 & 61,9 \\
\hline Tontura (labirintite / vertigens) & 39 & 40,2 \\
\hline Doença Vascular Periférica & 30 & 30,9 \\
\hline Diabetes Mellitus & 27 & 27,8 \\
\hline Artrite & 26 & 26,8 \\
\hline Doença gastrintestinal & 26 & 26,8 \\
\hline Insônia & 21 & 21,6 \\
\hline Incontinência Urinária ou Fecal & 18 & 18,6 \\
\hline Osteoporose & 17 & 17,5 \\
\hline Audição prejudicada & 13 & 13,4 \\
\hline Doença cardíaca & 13 & 13,4 \\
\hline Ansiedade/transtorno do pânico & 12 & 12,4 \\
\hline Asma & 11 & 11,3 \\
\hline Acidente Vascular Cerebral & 10 & 10,3 \\
\hline Obesidade & 9 & 9,3 \\
\hline Câncer & 6 & 6,2 \\
\hline DPOC / enfisema & 6 & 6,2 \\
\hline Constipação & 5 & 5,2 \\
\hline Anemia & 4 & 4,1 \\
\hline Doença neurológica & 3 & 3,1 \\
\hline
\end{tabular}

Fonte: Dados da pesquisa

Prejuízos na visão, hipertensão arterial sistêmica e problemas de coluna, foram as enfermidades mais referidas pelos idosos, tendo, cada uma delas, sido relatada por $60(61,9 \%)$ participantes. Outras doenças de significativa importância para o estudo e que demonstraram prevalência foram tontura (labirintite/vertigem) com
$39(40,2 \%)$ casos, doença vascular periférica, com 30 (30,9\%), diabetes mellitus $27(27,8 \%)$, artrite $26(26,8 \%)$ e osteoporose com 17 (17,5\%) casos.

Sobre a investigação de quedas, 32 idosos referiram ter caído pelo menos uma vez nos últimos 12 meses, estimando prevalência de 33\% (IC 95\%: 24,2\% - 42,8\%) de quedas entre os participantes. $\mathrm{O}$ número de quedas variou de uma a 10 vezes no último ano, com média de duas quedas por idoso ( \pm 2 quedas). Todos os idosos afirmaram que não fizeram uso de bebida alcoólica antes da ocorrência da queda.

A tabela 3 apresenta as características relacionadas à última queda dos idosos. Quanto aos tipos de queda, $28(87,5 \%)$ relataram ter caído da própria altura. As principais causas apontadas foram pisos escorregadios/ molhados, tontura/vertigem e perda da rigidez do corpo sem perda de consciência, com ocorrência de 7 (21,9\%), $4(12,5 \%)$ e $4(12,5 \%)$ casos, respectivamente. No que diz respeito ao local da queda, rua/avenida foi o espaço de maior ocorrência com 10 (31,3\%) casos, seguido de cozinha e ball de entrada, com o número de 5 (15,6\%) e $4(12,5 \%)$ nesta ordem. Ainda foi questionado o tipo de vestimenta que os pacientes usavam no momento da que$\mathrm{da}$, sendo o uso de calçados inadequados relatado por 22 $(68,8 \%)$ idosos.

Tabela 3. Características relacionadas à última queda sofrida pelos idosos atendidos na unidade de atenção primária de um município do estado de Goiás, 2015

(continua)

\begin{tabular}{lcc}
\hline \multicolumn{1}{c}{ Características da queda } & N & \% \\
\hline Tipo de queda & & \\
\hline Própria altura & 28 & 87,5 \\
Escada & 2 & 6,3 \\
Cadeira/poltrona & 1 & 3,1 \\
Cama & 1 & 3,1 \\
\hline Causa & & \\
\hline Dificuldade ao caminhar & 3 & 9,4 \\
Alterações de equilíbrio & 3 & 9,4 \\
Tontura/Vertigem & 4 & 12,5 \\
Perda da rigidez do corpo sem perda de & 4 & 12,5 \\
consciência & & \\
Tapetes soltos & 1 & 3,1 \\
Pisos irregulares/com buracos & 2 & 6,3 \\
Pisos escorregadios/molhados & 7 & 21,9 \\
Degrau alto/desnível no piso & 2 & 6,3 \\
\hline
\end{tabular}


(conclusão)

\begin{tabular}{lcc}
\hline \multicolumn{1}{c}{ Características da queda } & N & $\%$ \\
\hline Subir em objeto móvel para alcançar algo & 3 & 9,4 \\
Outros & 3 & 9,4 \\
\hline Local da queda & & \\
\hline Pátio/quintal & 2 & 6,3 \\
Cozinha & 5 & 15,6 \\
Hall de entrada & 4 & 12,5 \\
Dormitório & 3 & 9,4 \\
Sala & 1 & 3,1 \\
Banheiro & 2 & 6,3 \\
Calçada & 4 & 12,5 \\
Rua/avenida & 10 & 31,3 \\
Fazenda & 1 & 3,1 \\
\hline
\end{tabular}

Vestimentas que contribuíram para a

queda

\begin{tabular}{lcc}
\hline Calçados inadequados & 22 & 68,8 \\
Acessórios de apoio & 1 & 3,1 \\
Não se aplica & 9 & 28,1 \\
\hline Total & $\mathbf{3 2}$ & $\mathbf{1 0 0}$ \\
\hline
\end{tabular}

Fonte: Dados da pesquisa

Quando questionados sobre as consequências imediatas e permanentes da queda, 9 (28,1\%) idosos referiram a necessidade de hospitalização, com 3 (9,4\%) deles exigindo ainda intervenção cirúrgica por ocasião de fraturas ósseas em membros superiores e quadril (Tabela 4).

Tabela 4. Consequências da queda sofrida pelos idosos atendidos na unidade de atenção primária de um município do estado de Goiás, 2015

(continua)

\begin{tabular}{lcc}
\hline \multicolumn{1}{c}{ Consequências } & N & $\%$ \\
\hline Hospitalização & 9 & 28,1 \\
\hline Intervenção cirúrgica & 3 & 9,4 \\
\hline Fratura em MSS & 2 & 6,3 \\
Fratura em quadril & 1 & 3,1 \\
\hline Consequências imediatas à queda & & \\
\hline Ferimento com ponto & 1 & 3,1 \\
Entorse/uxação & 4 & 12,5 \\
Fratura fechada & 3 & 9,4 \\
Escoriações & 7 & 21,9 \\
Nenhuma consequência imediata & 17 & 53,1 \\
\hline Consequências permanentes da queda & & \\
\hline Afetou o andar & 12 & 37,5 \\
\hline
\end{tabular}

\begin{tabular}{lcc} 
& \multicolumn{2}{c}{ (conclusão) } \\
\hline \multicolumn{1}{c}{ Consequências } & N & \% \\
\hline Precisa de ajuda para as AVDs & 2 & 6,3 \\
Medo de cair novamente & 16 & 50,0 \\
Depressão/isolamento & 2 & 6,3 \\
Ansiedade & 3 & 9,4 \\
Perda da autonomia & 2 & 6,3 \\
\hline Fonte: Dados da pesquisa & \multicolumn{2}{c}{}
\end{tabular}

Mais de $50 \%$ dos casos de queda não tiveram consequências imediatas, porém em 7 (21,9\%) causaram escoriações, 4 (12,5\%) entorses/luxações, 3 (9,4\%) fraturas fechadas e $1(3,1 \%)$ ferimento com ponto. Em relação às consequências permanentes da queda, 16 (50\%) afirmaram temer cair novamente, 12 (37,5\%) relataram que a queda afetou o andar, e outros 9 (28,3\%) casos apontaram repercussões como: necessidade de ajuda para as atividades de vida diária (AVDs), perda da autonomia, ansiedade ou depressão/isolamento.

\section{DISCUSSÃO}

A prevalência de quedas observada entre os idosos deste estudo foi de 33\%, corroborando com o mencionado pelo Ministério da Saúde do Brasil, no ano de 2007 para a população idosa brasileira (30\%), assim como compatível com outros estudos nacionais (SIQUEIRA et al., 2011; CRUZ et al., 2012; CUNHA; LOURENÇO, 2014) e internacionais (BEKIBELE; GUREJE, 2010; ORCES, 2013).

Entre os participantes do estudo, houve predomínio do sexo feminino, com idade entre 60 e 69 anos, e que estudaram apenas até o nível primário (SIQUEIRA et al., 2007; REZENDE; CARRILLO; SEBASTIÃO, 2012; ARAÚJO et al., 2014). Este fato está relacionado com a crescente proporção do sexo feminino em relação ao masculino, podendo ser explicada pela melhor qualidade de vida que as mulheres adquirem e pela maior taxa de mortalidade sofrida pelo sexo masculino (PINHO et al., 2012).

No tocante aos hábitos de vida, a maioria dos idosos não praticava atividade física regular. Essa informação é de grande relevância, uma vez que o processo de envelhecimento provoca transformações no sistema 
sensitivo-motor, causando instabilidade postural, o que pode predispor o indivíduo a quedas. Nesse sentido, a prática de exercício físico poderia trazer benefícios aos idosos desse estudo, visto que é capaz de favorecer a mobilidade física e a estabilidade postural, associações suscetíveis à minimização do número de quedas (TOMICKI et al., 2016).

A maioria dos idosos relatou pelo menos um problema de saúde, sendo as alterações visuais, hipertensão arterial sistêmica e os problemas de coluna, as principais queixas. As alterações visuais são um dos mais importantes fatores de risco para quedas, uma vez que há distúrbio na relação de profundidade e na sensibilidade de identificar contrastes, além da diminuição da acuidade visual, dificultando a percepção de uma barreira (OLIVEIRA et al., 2014).

Os achados deste estudo demonstraram que cair da própria altura e por fatores relacionados ao ambiente inadequado, foram as características mais citadas entre os idosos em relação às quedas sofridas, corroborando com outros estudos da literatura (PINHO et al., 2012; OLIVEIRA et al., 2014).

As causas das quedas entre os idosos são múltiplas, podendo ou não estar associadas. Os fatores de risco para quedas são classificados em intrínsecos e extrínsecos. Os intrínsecos referem-se às alterações normais do processo de envelhecimento, circunstâncias de patologias e consequência do uso de fármacos. Já os fatores extrínsecos são os condicionantes sociais e ambientais os quais os idosos são expostos (ALMEIDA et al., 2012).

Neste estudo os fatores intrínsecos fisiológicos apontados como possíveis causas de queda foram dificuldade de caminhar, alterações do equilíbrio e perda da rigidez do corpo. Em relação à dificuldade de caminhar, no envelhecimento a deambulação sofre alterações, podendo o idoso apresentar uma redução no comprimento e altura das passadas possibilitando o risco de tropeços e quedas (OLIVEIRA et al., 2014).

As alterações do equilíbrio estão intimamente relacionadas com a redução da força muscular e aumento do tempo de reação, o que eleva a possibilidade de quedas frente a uma barreira (ALBINO et al., 2012; FECHINE; TROMPIERI, 2012; OLIVEIRA et al., 2014). Por fim, a perda de rigidez do corpo também está associada à perda de força muscular, que é comprometida pela diminuição do comprimento, elasticidade e número de fibras musculares; e pelo aumento da rigidez dos tendões e ligamentos (FECHINE; TROMPIERI, 2012).

No que tange aos fatores extrínsecos, a presença de pisos escorregadios/molhados foi a causa de maior prevalência neste estudo, podendo ser explicada pela falta de atenção que o idoso passa a ter em seu domicílio, uma vez que há familiaridade e autoconfiança no ambiente conhecido, sendo a cozinha, hall de entrada e dormitório os lugares com maior número de quedas no que diz respeito ao local onde a queda ocorreu (BORGES; MARINHO FILHO; MASCARENHAS, 2010). Subir em objetos móveis para alcançar algo mais alto também foi um fator extrínseco importante apontado neste estudo. Sugere-se que tal fato aconteça porque uma boa parte dos idosos reside sozinhos, levando-os a fazer suas atividades de vida diária sem auxílio e com uma segurança menor.

Ainda em relação ao local onde a queda ocorreu observou-se que rua/avenida foi onde se obteve o maior número de quedas fora do domicílio. Idosos com saúde preservada estão mais expostos a fatores ambientais externos, como ruas, calçadas e meios-fios, o que aumenta o risco de quedas. Contudo, aqueles que apresentam a saúde comprometida estão mais sujeitos a fatores intrínsecos, ou seja, as próprias limitações os colocam em vulnerabilidade às quedas (OLIVEIRA et al., 2014). Outro item importante no tocante aos fatores extrínsecos, diz respeito à utilização de calçados inadequados, onde mais da metade dos idosos que referiram queda afirmaram utilizar, o que implica em maior risco, principalmente quando o ambiente é impróprio.

A hospitalização foi uma consequência importante decorrente das quedas entre os idosos deste estudo. Para estes longevos permanecer acamado limita os movimentos levando a implicações físicas, assim como impactos psicológicos, provocando estresse emocional, dependência dos serviços hospitalares e suspensão de suas atividades corriqueiras (SANTOS et al., 2013). Além disso, a partir da ideia de hospitalização, as quedas geram impacto na saúde pública e privada e os custos arcados pelos governos são altos, o que aponta a necessidade de medidas públicas para a prevenção de quedas (AMBROSE; PAUL; HAUSDORFF, 2013).

As quedas não causaram, na maioria dos idosos, consequências imediatas. Contudo, as mais relatadas nes- 
te estudo foram as escoriações, dado semelhante aos de outras pesquisas (FERRETTI; LUNARDI; BRUSHI, 2013).

Diferentemente de outros estudos, os membros superiores foram os locais de maior ocorrência de fraturas. Presume-se que esta realidade seja decorrente da forma que os idosos tenham caído, tendo utilizado os membros superiores para apoio e proteção no momento da queda, proporcionando uma maior força sobre estes membros. Outra estrutura óssea referida como local de fratura, e já bem descrita na literatura, foi o quadril (ARAÚJO et al., 2014; BAKKEN et al., 2013; DEL DUCA; ANTES; HALLAL, 2013).

No que diz respeito às consequências permanentes da queda várias foram as queixas, sendo o medo de cair novamente, o mais referido, corroborando com o estudo de Santos et al. (2015). Este sentimento pode acarretar alterações psicossociais e emocionais nos idosos, como perda da autonomia e independência, incapacidade de realizar suas atividades de vida diária (AVD) e instrumentais de vida diária (AIVD), além de isolamento social e ideia de fragilidade e insegurança (ANTES et al., 2013).

Outra consequência permanente que a queda trouxe aos idosos foi a modificação do padrão de marcha, sendo esta uma importante informação na análise das AVDs, podendo gerar dependência desses longevos no quadro pós-queda. Embora não se conheça a gênese dessa sequela, supõe-se que, por mediação de lesões ósseas e ligamentares, o andar tenha sido afetado, até porque, no caso de terem permanecido imobilizados, atrofia muscular significativa pode ter ocorrido, causando alteração na deambulação. Os distúrbios de marcha (diminuição da velocidade, redução no comprimento do passo e redução na largura do passo) estão associados com a perda de independência e qualidade de vida (ZIDAN et al., 2012).

\section{CONCLUSÃO}

Os resultados deste estudo chamam à atenção sobre a situação em que se encontram os idosos residentes em uma comunidade do município de Aparecida de Goiânia, quanto à prevalência de quedas, e suscitam desafios aos profissionais de saúde atuantes na atenção primária.
No entanto, estes mesmos resultados devem ser apreciados considerando algumas limitações do estudo, tais como ter sido realizado em uma única unidade de saúde, com amostra pequena, o que traz restrições na generalização dos achados. Por se tratar de estudo transversal, há possibilidade de viés de memória e limite das conclusões sobre a direção ou causalidade das associações observadas.

A prevalência de quedas apresentou índices importantes, trazendo repercussões prejudiciais à saúde dos idosos. Diante disso, ressalta-se a necessidade de maior atuação dos profissionais de saúde na prevenção de quedas, por meio de ações integrais, com vistas à contribuir para a qualidade de vida dos longevos e diminuir as consequências negativas deste evento sobre sua saúde.

\section{REFERÊNCIAS}

ALBINO, I. L. R.; FREITAS, C. R.; TEIXEIRA, A. R.; GONÇALVES, A. K.; SANTOS, A. M. P. V.; BÓS, A. J. G. Influência do treinamento de força muscular e de flexibilidade articular sobre o equilíbrio corporal em idosas. Rev. bras. geriatr. gerontol, v. 15, n. 1, p. 17-25, 2012.

ALMEIDA, S. T.; SOLDERA, C. L. C.; CARLI, G. A.; GOMES, I.; RESENDE, T. L. Analysis of extrinsic a,nd intrinsic factors that predispose elderly individuals to fall. Rev Assoc Med Bras., v. 58, n. 4, p. 427-33, 2012.

AMBROSE, A. F.; PAUL, G.; HAUSDORFF, J. M. Risk factors for falls among older adults: a review of the literature. Maturitas, v. 75, n. 1, p. 51-61, 2013.

ANTES, D. L.; SCHNEIDER, I. J. C.; BENEDETTI, T. R.; D'ORSI, E. Fear of recurrent falls and associated factors among older adults from Florianópolis, Santa Catarina State, Brazil. Cad. Saúde Pública, v. 29, n. 4, p. 758-68, abr. 2013.

ARAÚJO, S. P.; MAIA, J. R. P.; VIEIRA, J. N. L.; SOARES, K. V. B. C.; DIAS, R. S. Fall characteristics and observations in São Luís elderly residents, Maranhão, Brazil. Rev Pesq Saúde, v. 15, n. 3, p. 331-5, 2014.

BAKKEN, M. S.; ENGELAND, A.; ENGESAETER, L. B.; RANHOFF, A. H.; HUNSKAAR, S.; RUTHS, S. Increased risk 
of hip fracture among older people using antidepressant drugs: data from the Norwegian Prescription Database and the Norwegian Hip Fracture Registry. Age and Ageing, v. 1, p. 1-6, 2013.

BEKIBELE, C. O.; GUREJE, O. Fall incidence in a population of elderly persons in Nigeria. Gerontology, v. 56, n. 3, p. 278-283, 2010.

BORGES, P. S.; MARINHO FILHO, L. E. N.; MASCARENHAS, C. H. M. Correlação entre equilíbrio e ambiente domiciliar como risco de quedas em idosos com acidente vascular encefálico. Rev. bras. geriatr. gerontol., v. 13, n. 1, p. 41-50, 2010.

CUNHA, A. A.; LOURENÇO, R. A. Quedas em idosos: prevalência e fatores associados. Revista HUPE, Rio de Janeiro, v. 13, n. 2, p. 21-29, 2014.

CRUZ, D. T.; RIBEIRO, L. C.; VIEIRA, M. T.; TEIXEIRA, M. T. B.; BASTOS, R. R.; LEITE, I. C. G. Prevalence of falls and associated factors in elderly individuals. Rev Saúde Pública, v. 46, n. 1, p. 138-146, 2012.

DEL DUCA, G. F.; ANTES, D. L.; HALLAL, P. C. Falls and fractures among older adults living in long-term care. Rev Bras Epidemiol., v. 16, n. 1, p. 68-76, 2013.

FERRETTI, F.; LUNARDI, D.; BRUSHI, L. Causes and consequences of falls in elderly at home. Fisioter Mov., v. 26, n. 4, p. 753-762, 2013.

FECHINE, B. R. A.; TROMPIERI, N. O processo de envelhecimento: as principais alterações que acontecem com o idoso com o passar dos anos. Rev Cientif Internacional., v. 1, n. 20, p. 106-132, 2012.

FHON, J. R. S.; ROSSEL, I.; FREITAS, C. P.; et al. Prevalência de quedas de idosos em situação de fragilidade. Rev. Saúde Pública, v. 47, n. 2, p. 266-273, 2013.

OLIVEIRA, A. D.; TREVANZI, P. F.; BESTETTI, M. L. T.; MELO, R. C. Fatores ambientais e risco de quedas em idosos: revisão sistemática. Rev. bras. geriatr. gerontol., v. 17, n. 3, p. 637-645, 2014.

ORCES, C. H. Prevalence and determinants of falls among older adults in Ecuador: na analysis of the SABE I Survey. Curr Gerontol Geriatr Res, v. 2013, fev. 2013.
PALMA, C. M. T. S. Quedas nos idosos: do risco à prevenção. 2012. 94 f. Dissertação (Mestrado em Enfermagem em Saúde Comunitária. Instituto Politécnico de Beja, Escola Superior de Saúde, Beja, 2012.

PINHO, T. A. M.; SILVA, A. O.; TURA, L. F. R.; MOREIRA, M. A. S. P.; GURGEL, S. N.; SMITH, A. A. F.; BEZERRA, V. P. Avaliação do risco de quedas em idosos atendidos em unidade básica de saúde. Rev. esc. enferm. USP, v. 46, n. 2, p. 320-327, 2012.

REZENDE, C. P.; CARRILLO, M. C. G. G.; SEBASTIÃO, E. C. O. Queda entre idosos no Brasil e sua relação com o uso de medicamentos: revisão sistemática. Cad. Saúde Pública, Rio de Janeiro, v. 28, n. 12, p. 2223-2235, 2012.

SANTOS, E. C. C.; BARBOSA, N. C.; MEDEIROS, J. D.; GRANJA, K. S. B.; CONSTANT, M. H. L.; CALLES, A. C. N. Declínio da capacidade de independência funcional em indivíduos idosos hospitalizados. Ciências Biológicas e de Saúde, v. 1, n. 3, p. 91-100, 2013.

SANTOS, R. K. M.; MACIEL, A. C. C.; BRITTO, H. M. J. S.; LIMA, J. C. C.; SOUZA, T. O. Prevalência e fatores associados ao risco de quedas em idosos adscritos a uma Unidade Básica de Saúde do município de Natal, RN, Brasil. Revista Ciência \& Saúde Coletiva, v. 20, n. 12, p. 3753-3762, 2015.

SHIAVETO, F. V. Avaliação do risco de quedas em idosos na comunidade. 2008. 118 fls. Dissertação (Mestrado em Enfermagem) - Universidade de São Paulo, Ribeirão Preto, 2008.

SIQUEIRA, F. V.; FACCHINI, L. A.; PICCINI, R. X.; TOMASI, E.; THUMÉ, E.; SILVEIRA, D. S.; VIEIRA, V.; HALLAL, P. C. Prevalência de quedas em idosos e fatores associados. Rev. Saúde Pública, v. 41, n. 5, p. 749-756, 2007.

SIQUEIRA, F. V.; FACCHINI, L. A.; SILVEIRA, D. S.; PICCINI, R. X.; TOMASI, E.; THUMÉ, E.; SILVA, S. M.; DILÉLIO, A. Prevalence of falls in elderly in Brazil: a countrywide analysis. Cad Saúde Pública, v. 27, n. 9, p. 1819-1826, 2011.

TOMICKI, C.; ZANINI, S. C. C.; CECCHIN, L.; et al. Effect of physical exercise program on the balance and risk of falls of institucionalized elderly persons: a randomized 
clinical trial. Rev. bras. geriatr. gerontol., v. 19, n. 3, p. 473-482, maio/jun. 2016.

ZIDAN, M.; ARCOVERDE, C.; ARAÚJO, N. B.; VASQUES, P.; RIOS, A.; LAKS, J.; DESLANDES, A. Motor and functional changes in different stages of Alzheimer's disease.

Rev Psiq Clín, v. 39, n. 5, p. 161-165, 2012.

Recebido em: 13 de setembro de 2016 Aceito em: 28 de fevereiro de 2017 